\title{
Impact of Experiencing Specific Side-Effects on Contraceptive Switching and Discontinuation in Uganda: Results from a Longitudinal PMA Survey
}

Linnea A Zimmerman ( $\square$ linnea.zimmerman@jhu.edu )

https://orcid.org/0000-0002-0118-0889

\section{Dana Sarnak}

Johns Hopkins University Bloomberg School of Public Health

Celia Karp

Johns Hopkins University Bloomberg School of Public Health

Shannon Wood

Johns Hopkins University Bloomberg School of Public Health

Saifuddin Ahmed

Johns Hopkins University Bloomberg School of Public Health https://orcid.org/0000-0002-9884-4580

Fredrick Makumbi

Makerere University https://orcid.org/0000-0002-6460-7325

Simon Kibira

Makerere University https://orcid.org/0000-0002-7385-423X

\section{Research}

Keywords: Contraceptive side-effects, contraceptive continuation, contraceptive switching, longitudinal, menstrual bleeding, sexual experience, Uganda

Posted Date: July 24th, 2020

DOI: https://doi.org/10.21203/rs.3.rs-47447/v1

License: (c) (i) This work is licensed under a Creative Commons Attribution 4.0 International License.

Read Full License 


\section{Abstract}

There is substantial evidence that contraceptive side effects are a major deterrent to consistent use of contraception but few studies in low- or middle-income countries explore the role that specific sideeffects, rather than the general category of "side-effects and health concerns", have on contraceptive dynamics. This study used population-based, longitudinal data to explore the effect that specific sideeffects had on contraceptive continuation, discontinuation, and switching in Uganda.

Methods Data for this study come from two rounds of PMA2020 data collection in Uganda PMA2020 Uganda's sixth cross-sectional survey and a follow-up survey conducted one year later. The main outcomes of interest were discontinuation and switching among modern users of hormonal contraceptive methods at baseline $(n=550)$. Multivariable multinomial logistic regressions assessed the association of reporting experiencing specific side effects (more bleeding, less bleeding, irregular bleeding, changes to sexual experience, and physical discomfort) with discontinuation and switching one year later, adjusting for socio-demographic characteristics, type of method, and length of use.

Results One-third of hormonal contraceptive users reported experiencing side-effects at baseline. Reporting more bleeding increased the risk of discontinuation by 2.39 times (95\% Cl: 0.01-4.72). Experiencing irregular bleeding decreased the risk of switching by a factor of 0.40 (95\% Cl: $0.17-$

0.93). Changes to sexual experience or physical discomfort were both associated with increased relative risk of switching; reporting a change to sexual experience increased the relative risk of switching by a factor of 3.10 (95\% Cl : 0.97-9.10) and physical discomfort increased the relative risk by a factor of 1.75 (95\% Cl: 0.95-3.24). Wealth was significantly negatively associated with discontinuation while education was marginally significantly and positively associated with switching.

Conclusions Greater attention should be paid to understanding the unique contributions of side effects to contraceptive behavior using population-based data. Not all side effects are associated with contraceptive discontinuation and switching, despite the experience of side effects being common. The experience of physical discomfort and changes to sexual experience, largely ignored in other research, were shown to affect use. Providing greater individualized care that includes information about common side effects and how they may impact daily life is necessary.

\section{Plain English Summary}

Research has shown that experiencing side effects is related to stopping use of contraception, even when women wish to avoid pregnancy. Most research, however, does not differentiate between different side effects, such as increased bleeding or changes to sexual pleasure, instead combining all into "side effects or health concerns. We used data from 550 women in Uganda, who were interviewed twice, one year apart, to see if women who reported different side-effects at the first interview were more likely to stop using contraception or switch to a different contraceptive method than women who did not report experiencing side effects. We found that increased menstrual bleeding was associated with a higher rate of contraceptive discontinuation, while women who reported that they had changes to their sexual 
experience or who reported physical discomfort, such as cramping, were more likely to switch methods than women who did not report these issues. It is important to understand what side effects are likely to motivate stopping versus switching contraception so that education and counseling can inform women of side effects they may experience, help them choose the best method based on what side effects they deem important, and if necessary, aid in switching contraceptive methods.

\section{Background}

There is substantial evidence that contraceptive side effects, either experienced or perceived, are a major deterrent to consistent use of contraception. The majority of women who discontinue contraception while still wanting to delay or avoid pregnancy do so for "method-related concerns" (1,2); Bradley and colleagues found that among women who discontinued while still in need of a method, as many as $37 \%$ reported side effects as their primary reason for discontinuation. Though a range of side effects associated with hormonal contraceptive use are known, relatively little research exists on the impact of specific forms of side effects, rather than the general category of "side-effects and health concerns", have on contraceptive continuation.

Prospective studies and clinical trials have documented a number of side effects arising from hormonal contraceptives and the non-hormonal copper IUD: disruptions to menstrual bleeding, breast tenderness, nausea, acne, headaches, dizziness, and changes in sexual desire (3-10). Though the wide range of side effects is well established, there has only recently been an effort to understand how each of these side effects are related to overall contraceptive use dynamics - adoption, discontinuation, and switching. Changes to menstrual bleeding, including increased bleeding, amenorrhea, and irregular bleeding and spotting, have been shown to be particularly influential on contraceptive use $(1,8,11)$. A recent review conducted by Wood and colleagues highlighted the critical dearth of population-based estimates regarding the extent that women experience contraceptive-induced changes in sexual experience in LMICs (12). Similar gaps in knowledge exist around the prevalence and consequences of other specific side effects in similar contexts. None of the population-based national surveys that constitute the majority of publicly available data on family planning, such as the DHS, PMA2020, and MICS, include questions that identify specific side effects, instead identifying only "side effects" or "health concerns" as a reason for contraceptive discontinuation (13-15). This limits our understanding of both the prevalence and effect of specific contraceptive induced side effects on contraceptive use behavior.

The majority of research that has highlighted the importance of specific side-effects in LMICs has been qualitative. Across diverse settings, women report experiencing changes to menstrual bleeding, abdominal pain, weight gain, changes to sexual experience, and non-specific "weakness" as influencing their decision-making about contraception $(13,16-22)$. Though providing critical contextual data, qualitative data are unable to assess how widespread experiences are, how these experiences contribute to contraceptive use dynamics at a population level, and what side effects may be largely tolerated by women versus those that lead to discontinuation or switching. 
Tolerability of side effects, that is, whether women continue using a method in the presence of side effects, is particularly relevant to consider in the context of LMIC settings. The majority of research on the influence of specific side effects on contraceptive behavior has been limited to high-income settings or conducted as part of clinical trials $(3,6,7,23-25)$. Additionally, while research has highlighted the role that contraceptive counseling can play in improving tolerability of side effects, most of these studies have been done in high- and middle-income settings (26-28). Though valuable, these findings are limited in their generalizability to low-income countries, where tolerability of specific side effects may differ for a variety of social, cultural, and economic reasons $(17,29)$. Schwarz and colleagues highlight that the experience of side effects and how these side effects are interpreted and tolerated is heavily influenced by context (17). The strength of pronatalist beliefs, gendered power dynamics, and individual motivation and circumstance are critical to understanding the embodied experiences of women. All vary significantly between high and low-income countries and across low-income settings; findings from high-income countries cannot thus be applied to low-income country contexts nor can findings from one low-income country be rotely applied to another. As Abdel-Tawab and RamaRao discuss, improvements to contraceptive services, which should address these embodied experiences, must be informed by context and modified to be contextually appropriate (30). Gaining a more nuanced understanding of the characteristics of women in specific settings who are able and willing to tolerate side effects and continue using contraception, versus those that discontinue, may thus provide valuable lessons to program managers and health providers.

Finally, limited research has explored how the experience of specific side effects may motivate switching to a different method versus discontinuing contraception altogether. Discontinuation and switching to a lesser effective method expose women to higher risk of unwanted or accidental pregnancies. The decision to stop using contraception versus switch to another method when experiencing side effects is likely informed by personal characteristics, such as parity or access to resources to manage side effects, in addition to motivation to delay pregnancy $(22,31)$. To our knowledge, the only prospective study in LMICs to assess specific side effects and their effect on contraceptive switching versus discontinuation was conducted by Barden-O'Fallon and colleagues among women in Honduras (31). The authors found that Honduran women who experienced amenorrhea or heavy menstrual bleeding were significantly more likely to discontinue than switch methods and that urban women were significantly more likely to switch methods than rural women, underscoring the simultaneous roles of side-effects and sociodemographic characteristics on women's contraceptive practices. However, the study did not compare switching and discontinuing separately to assess the effect of experiencing side effects on these behaviors, relative to continuation. Despite the logical contribution of side effects to women's decisions to adopt a different method or stop using contraception entirely, there is a clear dearth of research in this area.

To better understand the prevalence of different side-effects and their association with contraceptive use dynamics, a longitudinal study was conducted in Uganda. This study aimed to estimate the prevalence of a range of specific side effects that were experienced among users of hormonal contraception and to establish the association between these side-effects and contraceptive discontinuation, switching, or 
continuation one year later. Additionally, we sought to identify relevant socio-demographic characteristics associated with discontinuation and with switching.

\section{Methods}

\section{Study Overview and Sampling}

Data for this study come from two rounds of Performance Monitoring and Accountability (PMA2020) data collection in Uganda. Specifically, in PMA2020 Uganda's sixth cross-sectional survey (15), conducted from April to June 2018 (hereafter referred to as baseline), the consent and design were modified to enroll women in a longitudinal study. A follow-up survey was conducted approximately after one-year from May to June 2019.

The PMA2020 is a multi-stage cluster, nationally representative survey of women age $15-49$. In the baseline survey, 110 Enumeration Areas (EA) were selected using probability proportional to size sampling and all occupied households were enumerated. Forty-four households were randomly selected within each EA, consented, and interviewed. All women age 15-49 who were either usual members of the household or who slept in the household the night before were approached for interview, and if consented, interviewed by a trained interviewer. A total sample of 4,288 women were interviewed in the baseline survey; the majority $(95.5 \% ; n=4,095)$ of women agreed to participate in the follow-up survey. Further information on the design of PMA2020 surveys is available from www.pmadata.org and Zimmerman et al (2017).

At follow-up, interviewers returned to the households of women who completed the baseline survey and re-consented women to participate in the follow-up survey. We were able to relocate and successfully interview 2,755 of the original sample, resulting in a follow-up rate of $67 \%$. Due to potential bias from loss to follow-up, we constructed an inverse probability weight from estimated propensity scores to adjust differential loss to follow-up. The probability weight of loss to follow-up was then multiplied by the original baseline individual female weight to construct the final weight in the analyses. We fit a multivariable multinomial logistic regression model with women's age, parity, fertility intention, marital status, education (years of schooling), wealth quintile and residence as covariates. No significant differences in baseline characteristics between all women in the baseline and those who were successfully followed-up were detected after the application of the final sampling weights.

\section{Analytic Sample}

This analysis was restricted to women who reported currently using hormonal contraception (implant, injectable, pill) and IUD at baseline. Users of emergency contraception were excluded due to the periodicity in its use and female sterilization users were excluded as they would not be able to discontinue or switch methods. Condoms and other barrier and traditional methods were excluded, as systemic side effects were not anticipated from using these methods. In total, 550 women were included 
in this analysis; the flowchart of sample selection process is shown in Fig. 1. Data was complete for all observations used in analysis.

\section{Measures}

At baseline, women were asked to report whether they were currently experiencing any side effects, and if so, to specify all that they were currently experiencing (with multiple-response options; responses were not read aloud). The list of side effects was drawn from the literature on clinically documented side effects, and those that have been reported in qualitative research in Uganda. The list was reviewed with the incountry team and pilot tested; the complete list of side-effects and frequencies with which they were reported is shown in Appendix 1.

The outcomes related to contraceptive behavior were defined based on the woman's contraceptive use status at the time of follow-up survey. Specifically, women were categorized in to three groups: either continuing (using the same method at baseline and follow-up), switched (using a different method at baseline and follow-up), or discontinued (not using a method at follow-up). We assessed the distribution of each outcome by method type (implant, IUD, injectables, and oral pills).

\section{Covariates}

All analyses adjusted for the following socio-demographic variables measured at baseline: age (categorical variable of 15-24, 25-34, and 35 and above), marital status (binary variable indicating married/in-union), residence (urban or rural), education (categorical variable indicating none, primary, or secondary and above), parity (categorical variable of $0-1,2-3$, or $4+$ children), and fertility intentions (wanted child within two years, wanted child after two years, wanted no more children). Due to sample size limitations, we created a binary variable for wealth, indicating whether the respondent resided in a household in one of the lowest two wealth quintiles (0) or the wealthier three (1). To account for the fact that side effects may be more frequent when initiating a method and resolve over time, we included a binary variable indicating whether the woman started her method less than 12 months prior to the baseline interview. We used a 12-month time frame to align with previous research (31). Finally, we adjusted for method used at baseline with a binary variable indicating short-acting (pill and injectable) or long-acting method (IUD and implant).

\section{Analysis}

Exploratory analyses assessed the prevalence of each side effect for further modeling considerations. Based on sample size, the following side effects were combined: "uterine cramping", "cramping", "headache", "nausea”, and "weakness" ("physical discomfort"); "vaginal dryness" and "lowered libido" ("changes to sexual experience"); "spotting" and "irregular bleeding” ("irregular bleeding”).

Only side effects that were reported by a minimum of 20 women were included in models. Unadjusted multinomial models assessed the relative risk of discontinuing or switching, relative to continuing, among women who reported experiencing the side effect compared to women who did not. As women could experience multiple side effects at once, we ran adjusted multinomial models that included all side 
effects and the covariates listed above. Sensitivity analyses were conducted with the inclusion of only one side effect at a time and results were largely consistent (Appendix 2 and 3 ).

Descriptive analyses applied the survey weights described above. Due to small sample sizes and because weighting may increase variances and design-effects substantially, we tested the efficiency of the application of weights in the unadjusted multinomial regressions. The inefficiency index (32) of each unadjusted multinomial model assessing the relationship of each side effect ranged from $8-56 \%$ with a median of $30 \%$. To maximize efficiency, we did not apply the survey weights to the final unadjusted or adjusted multinomial models but did adjust for clustering. All analyses were conducted using Stata v16.0 (33). We used the STROBE cohort checklist when writing our report (34).

\section{Result}

The characteristics of women in the analytic sample are shown in Table 1. The average age of women in the sample was 29.3 and the majority of women (81.3\%) were married or in-union. One out of five users was nulliparous or primiparous and the vast majority (94.1\%) had attended at least some schooling. About $24 \%$ of current users reported wanting a child within two years. About half of women reported that they had been using the current hormonal contraceptive method for fewer than 12 months. The majority of women were using the injectable (51.95\%) followed by the implant (34.0\%). 
Table 1:

Sample characteristics of current hormonal contraceptive users at baseline

\begin{tabular}{|c|c|c|c|}
\hline & $\%$ & SE \\
\hline & & $\mathrm{N}=$ & 550 \\
\hline \multicolumn{2}{|l|}{ Age(mean) } & 29.3 & 0.4 \\
\hline \multicolumn{2}{|c|}{ Married or in-union } & 81.3 & 2.3 \\
\hline \multicolumn{2}{|l|}{ Urban } & 22.2 & 3.7 \\
\hline \multicolumn{4}{|l|}{ Parity } \\
\hline & $0-1$ & 19.1 & 2.4 \\
\hline & $2-3$ & 36.8 & 3.0 \\
\hline & $4+$ & 44.1 & 3.1 \\
\hline \multirow[t]{4}{*}{ Education } & $4+$ & & \\
\hline & None & 5.9 & 1.2 \\
\hline & Primary & 54.0 & 2.8 \\
\hline & O-level + & 40.0 & 3.0 \\
\hline \multicolumn{4}{|l|}{ Wealth } \\
\hline & Lowest $40 \%$ & 29.0 & 3.3 \\
\hline & Highest $60 \%$ & 71.0 & 3.3 \\
\hline \multicolumn{4}{|c|}{ Fertility desire } \\
\hline & Want in $<2$ year & 23.9 & 3.1 \\
\hline & Want in $2+$ year & 44.9 & 2.8 \\
\hline & Do not want anymore & 31.2 & 2.6 \\
\hline Using $<12 m$ & onths & 47.8 & 2.9 \\
\hline \multicolumn{4}{|l|}{ Method } \\
\hline & Implant & 34.0 & 2.8 \\
\hline & IUD & 5.7 & 1.3 \\
\hline & Injectable & 52.0 & 3.0 \\
\hline & Pill & 8.4 & 1.6 \\
\hline
\end{tabular}


Table 2 shows the percentage of women who continued the same method at follow-up interview, switched, or discontinued the method that they were using at baseline by method type; in total, $38.0 \%$ of users were using the same method at follow-up; $25.2 \%$ had switched methods; and $36.8 \%$ had discontinued use. Switching was highest among pill users, while discontinuation was highest among injectable users.

Table 2:

Contraceptive discontinuation, switching, and continuation by method used at baseline $(\mathrm{N}=550)$

\begin{tabular}{|lllll|}
\hline & Discontinued & Switched & Continued & Total \\
\hline & $\%$ & $\%$ & $\%$ & $\%$ \\
\hline Implants & 29.8 & 11.9 & 58.2 & 100.0 \\
\hline IUD & 30.8 & 8.2 & 61.0 & 100.0 \\
Injectables & 43.4 & 33.7 & 22.9 & 100.0 \\
Pill & 28.4 & 37.5 & 34.1 & 100.0 \\
\hline Total & 36.8 & 25.2 & 38.0 & 100.0 \\
\hline
\end{tabular}

Table 3 shows the frequencies of side effects experienced among all uses, and by short- versus longacting method users. About one-third of hormonal contraceptive users (35.3\%) reported experiencing at least one side effect at baseline. There were no significant differences in the percentage of side-effects experienced by short- versus long-acting users, except that a higher percentage of short-acting method users $(16.2 \%)$ reported less bleeding than long-acting method users $(6.8 \%)(p=.02)$. 
Table 3:

Side effects reported at baseline among current users of hormonal contraception, all and by method type $(\mathrm{N}=550)$

\begin{tabular}{|c|c|c|c|c|c|c|c|}
\hline & \multicolumn{2}{|c|}{$\begin{array}{l}\text { All } \\
\text { Methods }\end{array}$} & \multicolumn{2}{|c|}{$\begin{array}{l}\text { Short Acting } \\
\text { Methods }\end{array}$} & \multicolumn{2}{|c|}{$\begin{array}{l}\text { Long Acting } \\
\text { Methods }\end{array}$} & \multirow[t]{2}{*}{$\begin{array}{l}\mathrm{p}- \\
\text { value }\end{array}$} \\
\hline & $\mathrm{n}$ & $\%$ & $\mathrm{n}$ & $\%$ & $\mathrm{n}$ & $\%$ & \\
\hline Any side effect & 194 & 35.3 & 119 & 38.1 & 75 & 31.1 & 0.25 \\
\hline \multicolumn{8}{|l|}{ Menstrual changes } \\
\hline Less bleeding & 66 & 12.5 & 49 & 16.2 & 17 & 6.8 & 0.02 \\
\hline More bleeding & 53 & 8.9 & 27 & 7.1 & 26 & 11.6 & 0.15 \\
\hline Irregular bleeding & 51 & 9.3 & 31 & 10.1 & 20 & 8.2 & 0.60 \\
\hline \multicolumn{8}{|l|}{ Other bodily changes } \\
\hline $\begin{array}{l}\text { Changes to sexual } \\
\text { experience }\end{array}$ & 27 & 6.5 & 17 & 7.4 & 10 & 5.2 & 0.59 \\
\hline Physical discomfort & 88 & 16.3 & 52 & 16.3 & 36 & 16.4 & 0.99 \\
\hline
\end{tabular}

Table 4 below shows the unadjusted (crude) relative risk ratios (RRR) of discontinuing and switching relative to continuing. Experience of any side effect increased the relative risk of discontinuing about $55 \%$ (cRRR: 1.55; 95\% Cl: 1.1-2.20). While bleeding less and irregular bleeding were not associated with an increased relative risk of discontinuation or switching, bleeding more was associated with increased relative risk of discontinuation (cRRR: $2.01,95 \% \mathrm{Cl}$ : 1.00-4.03. Physical discomfort was marginally associated with an increased relative risk of discontinuing by a factor of 1.67 (95\% Cl: 0.96-2.90) and switching by a factor of 1.74 ( $95 \% \mathrm{Cl}$ : 0.94-3.22). Though not associated with discontinuation, changes to sexual experience was marginally associated with an increased relative risk of switching of 2.76 times (95\% Cl: 0.89-8.58). 
Table 4:

Crude risk ratios of contraceptive discontinuation and contraceptive switching relative to continuation by side effect

\begin{tabular}{|lccccccccc}
\hline & \multicolumn{3}{l}{ Discontinued } & \multicolumn{5}{l}{ Switched } \\
& RRR & $95 \% \mathrm{Cl}$ & & RRR & $95 \% \mathrm{Cl}$ & \\
\hline Any side effect & $\mathbf{1 . 5 5}$ & $\mathbf{1 . 1 0}$ & $\mathbf{-}$ & $\mathbf{2 . 2 0}$ & 1.29 & 0.79 & - & 2.09 \\
\hline Less bleeding & 1.44 & 0.86 & - & 2.43 & 1.24 & 0.65 & - & 2.36 \\
\hline More bleeding & $\mathbf{2 . 0 1}$ & $\mathbf{1 . 0 0}$ & $\mathbf{-}$ & $\mathbf{4 . 0 3}$ & 1.36 & 0.61 & - & 3.07 \\
\hline Irregular bleeding & 1.58 & 0.83 & - & 3.01 & 0.72 & 0.29 & - & 1.78 \\
\hline Change to sexual experience & 1.41 & 0.43 & - & 4.62 & 2.76 & 0.89 & - & 8.58 \\
\hline Physical discomfort & 1.67 & 0.96 & - & 2.90 & 1.74 & 0.94 & - & 3.22 \\
\hline Note: Bold indicates $p<.05$, italicized indicates & $p<.10$ & & & \\
\hline
\end{tabular}

After adjustment with demographic, socio-economic and potential confounding covariates, the association between experiencing each side effect and contraceptive switching or discontinuing at follow-up were largely consistent (Table 5 below). Reporting more bleeding increased the risk of discontinuation by 2.39 times ( $95 \% \mathrm{Cl}$ : 1.21-4.72). Though irregular bleeding was not statistically significantly associated with switching in the crude models, after adjustment, experiencing irregular bleeding decreased the risk of switching by a factor of 0.40 (95\% Cl: $0.17-0.93)$. Reporting changes to sexual experience and physical discomfort were both marginally associated with increased relative risk of switching; reporting a change to sexual experience increased the relative risk of switching by a factor of $3.10(95 \% \mathrm{Cl}: 0.97-9.90)$ and physical discomfort increased the relative risk by a factor of 1.75 (95\% Cl: 0.95-3.24).

The risks of discontinuing and switching were significantly higher among women using short-acting methods (aRRR: $3.90 ; 95 \% \mathrm{Cl}$ : 2.44-6.24 and aRRR: $6.31 ; 95 \% \mathrm{Cl}$ : 3.52-11.31, respectively) relative to women using a long-acting method. Wealth and fertility desires were significantly associated with discontinuation, with wealthier women significantly less likely to discontinue (aRRR: 0.57 ; $95 \%$ Cl: $0.34-$ 0.96). Relative to women who reported wanting a child within the next two years, women who did not want a child in the next two years (aRRR: $0.33 ; 95 \% \mathrm{Cl}$ : $0.16-0.67$ ) or who wanted no more children (aRRR: $0.21 ; 95 \% \mathrm{Cl}$ : 0.09-0.45) were significantly less likely to discontinue use one year later. Age and education were marginally associated with increased relative risk of switching, though having either primary or secondary education appeared to increase the risk of switching by approximately a factor of 3 (aRRR: 3.06; 95\% Cl: 0.89-10.50 and aRRR: 3.47, 95\% Cl: 0.99-12.13, respectively). Appendix 2 and 3 shows the unadjusted associations for all background characteristics, the adjusted associations before accounting for specific side effects, and models with each side effect modeled separately. Associations are consistent in direction and generally consistent in significance. 
Table 5:

Adjusted relative risk of discontinuing and switching after adjusting for relevant background characteristics.

\begin{tabular}{|c|c|c|c|c|c|c|c|c|}
\hline & \multicolumn{4}{|c|}{ Discontinuation } & \multicolumn{4}{|c|}{ Switching } \\
\hline & RRR & $95 \% \mathrm{Cl}$ & & & RRR & $95 \% \mathrm{Cl}$ & & \\
\hline \multicolumn{9}{|l|}{ Side effect } \\
\hline Less bleeding & 1.30 & 0.73 & - & 2.32 & 0.97 & 0.46 & - & 2.02 \\
\hline More bleeding & 2.39 & 1.21 & - & 4.72 & 1.40 & 0.52 & - & 3.78 \\
\hline Irregular bleeding & 1.03 & 0.48 & - & 2.21 & 0.40 & 0.17 & - & 0.93 \\
\hline Changes to sexual experience & 1.13 & 0.35 & - & 3.65 & 3.10 & 0.97 & - & 9.90 \\
\hline Physical discomfort & 1.59 & 0.85 & - & 2.96 & 1.75 & 0.95 & - & 3.24 \\
\hline Using $<12$ months & 1.24 & 0.73 & - & 2.08 & 1.56 & 0.85 & - & 2.86 \\
\hline Using short acting method & 3.90 & 2.44 & - & 6.24 & 6.31 & 3.52 & - & 11.31 \\
\hline \multicolumn{9}{|l|}{ Age: ref(15-24) } \\
\hline $25-34$ & 1.84 & 0.87 & - & 3.92 & 2.10 & 0.97 & - & 4.55 \\
\hline $35-49$ & 1.29 & 0.45 & - & 3.71 & 1.14 & 0.37 & - & 3.54 \\
\hline Married/ In-union & 0.54 & 0.28 & - & 1.01 & 0.63 & 0.28 & - & 1.40 \\
\hline Urban & 1.25 & 0.76 & - & 2.07 & 1.22 & 0.65 & - & 2.31 \\
\hline \multicolumn{9}{|l|}{ Parity: ref(0-1) } \\
\hline $2-3$ & 0.78 & 0.36 & - & 1.71 & 0.87 & 0.37 & - & 2.08 \\
\hline $4+$ & 0.78 & 0.28 & - & 2.15 & 1.27 & 0.36 & - & 4.48 \\
\hline \multicolumn{9}{|l|}{ Education: ref(None) } \\
\hline Primary & 0.84 & 0.36 & - & 1.99 & 3.06 & 0.89 & - & 10.50 \\
\hline Secondary + & 0.66 & 0.25 & - & 1.72 & 3.47 & 0.99 & - & 12.13 \\
\hline Wealth (ref: Lowest 40\%) & 0.57 & 0.34 & - & 0.96 & 0.62 & 0.35 & - & 1.11 \\
\hline \multicolumn{9}{|l|}{ Fertility desires: ref(Want <2 year) } \\
\hline Want in $2+$ year & 0.33 & 0.16 & - & 0.67 & 0.64 & 0.31 & - & 1.29 \\
\hline Do not want anymore & 0.21 & 0.09 & - & 0.45 & 0.39 & 0.14 & - & 1.04 \\
\hline
\end{tabular}

\section{Discussion}


We found that about one-third of women experienced side effects while using hormonal methods, and that their associations with switching and discontinuation varied substantially based on the nature of side-effects. Experiencing more bleeding was strongly associated with discontinuation, while irregular bleeding, physical discomfort, and changes to sexual experience were associated with discontinuation. These results underscore the importance of examining the unique influence of specific side effects, instead of broad singular category of "side effects and health concerns", in studies of contraceptive practices. Failure to investigate specific contraceptive side effects inhibits understanding of nuanced relationships between women's experiences and their adaptive responses to contraceptive behaviors.

Our finding that increased menstrual bleeding was associated with discontinuation aligns with a growing body of literature, highlighting the importance of contraceptive-induced bleeding changes $(1,11)$. Excessive bleeding arose as a major barrier to contraceptive use in previous qualitative research in Uganda $(22,35,36)$; that excessive bleeding was strongly associated with discontinuation, but not with switching, indicates that women who experience this side effect may be reluctant to switch to new methods, instead preferring to discontinue use altogether. The experience of excessive bleeding may be additionally problematic in environments like Uganda, where myths and misperceptions around the harmful impacts of contraception, including effects on future fertility and on overall health, are widespread $(22,37,38)$. In strongly pronatalist cultures such as Uganda, where concerns of fertility are paramount, excessive bleeding may not be viewed simply as an inconvenience, but as a prequel to infertility. This belief carries profound social consequences and, therefore, may greatly reduce motivation to use contraception $(17,39)$

We did not find significant effects with discontinuation or switching among women who reported less bleeding, although irregular bleeding was shown to reduce the risk of switching. Evidence from qualitative studies in Africa suggests that amenorrhea and irregular bleeding are often viewed negatively and associated with concerns about future fertility, particularly among nulliparous women (11), however recent qualitative evidence in Uganda found that the potential for lighter periods as a side effect of contraception was viewed favorably by women (36). Zimmerman and colleagues found that two-thirds of sexually active women in Uganda strongly agreed that not having a menstrual period as a result of contraceptive use was unhealthy, but agreement was not strongly associated with either discontinuation or switching (Under Review). This supports the current findings and suggests that while concerns about amenorrhea are prevalent, they did not seem to motivate contraceptive discontinuation in this sample. Of note, however, we did not distinguish between less bleeding and cessation of bleeding. Future research should attempt to distinguish between these two bleeding patterns, as complete cessation may engender different behavioral responses than a lighter, but consistent, menstrual period.

Though experiencing changes to sexual experience and physical discomfort were only marginally statistically significant, both were associated with increased risk of switching; changes to sexual experience demonstrated a particularly large effect size. One explanation for why these are related to switching rather than discontinuation is that these side effects may be perceived as less related to fertility risk; women may thus be willing to try another method, rather than discontinue use altogether. Very little 
research has explored either quantitatively or qualitatively how changes to sexual experience induced by contraceptive use affect contraceptive dynamics, despite evidence that changes to sexual experience and enjoyment for both the woman and her partner are critical $(12,20,22,40)$. Similarly, physical discomfort, which here included headache, nausea, weakness, and cramping, have generally not been included in studies that assess the impact of side effects. Unlike our findings, Barden-O'Fallon and colleagues found that abdominal cramping was not associated with the decision to switch versus stop method use. However, the authors did not assess whether cramping increased the overall risk of either relative to continuation (31). Some qualitative research in low-income countries has identified cramping as a concern (20), but the majority of research that has explored this side effect has been conducted in high income countries, thereby limiting comparison with countries like Uganda $(41,42)$. This limits our ability to compare our findings to other studies and underscores the critical need to explore these areas of contraceptive use and experience more fully.

A secondary objective of this paper was to gain a better understanding of the characteristics of women who continue, discontinue, and switch methods after experiencing side effects, in order to explore how life circumstances influence contraceptive decision-making. Due to sample size limitations, we could not limit our analyses to only women who reported experiencing a side effect, however, we were able to identify several relevant sociodemographic characteristics associated with discontinuation and switching, after adjusting for the experience of specific side effects. Women who wanted to delay or limit childbearing by more than two years were significantly less likely to discontinue use, but these same motivations had little relationship with switching; indicating that fertility desires impact whether a method is used but may have less influence on what method specifically is chosen. Qualitative evidence from Uganda highlights the significant role that motivation to avoid pregnancy plays on contraceptive continuation, even when negative side effects are experienced (22). Barden-O'Fallon found that fertility intentions did not differentiate switchers from discontinuers, which seems to contradict our findings, but less research has examined what role, if any, fertility intention may play on the decision to switch methods when side effects are experienced (31).

Interestingly, we also found that wealth was associated with decreased relative risk of discontinuation, while education was associated with an increased risk of switching, indicating that social privilege may influence contraceptive dynamics in different ways. For example, social disadvantages that inhibit poorer women from seeking healthcare services to adopt new methods, in the presence of side effects, may result in higher discontinuation among this population. In contrast, wealthier women who have greater access to contraceptive services, and perhaps enhanced counseling from providers, may be less likely to discontinue their methods. Additionally, educated women may be more knowledgeable about a range of contraceptive methods than less educated women, allowing them greater opportunity to switch methods if they are dissatisfied. Despite experience of side effects, education and knowledge may translate into use of preferred methods and continuation of these methods.

A potential limitation of our study is that women were asked to spontaneously report side effects that they were experiencing, rather than replying directly to a list of potential side effects. To do so, women 
needed to consider what they were experiencing as a side effect of their contraceptive method and, in the case of amenorrhea and loss of libido, recognize the absence or reduction of these experiences as being currently experienced. It is possible that women are less likely to report something that is not happening as being "currently experienced", relative to something that has increased or newly presented, but this requires further investigation. Reading a list of potential side effects and requiring a response may be a more effective way to gather information on prevalence of side effects, but may also result in overreporting $(43,44)$. Under-reporting, in addition to our lower than expected retention, may have contributed to our limited analytical sample size. The limited absolute numbers of women, particularly for switching, restricted our ability to explore relationships across the full range of side effects. However, even with our limited sample, one in three women in Uganda who were current users of hormonal contraceptive indicated that they were experiencing some form of side effects, underscoring how common these experiences are. Additionally, our retention at study level was $67 \%$, with higher attrition amongst certain sub-groups (reported in Zimmerman et al, Under Review). The application of propensity scores to adjust for loss-to-follow-up allowed us to construct weights that generated estimates of sample characteristics that did not differ between the full baseline and the sample of women who successfully completed follow-up. While we believe we thus adjusted for the majority of demographic and economic characteristics that may be related to contraceptive continuation, it is possible that there are unadjusted confounders that differ between these groups and may affect the overall generalizability of our findings. Finally, we defined switching and discontinuation only based on baseline and follow-up and were unable to account for events that occurred between the two time points. Though we included a contraceptive calendar for this purpose, we found that recall of starting and ending dates of methods when reported retrospectively was inconsistent and rendered the calendar data unusable for this purpose.

Despite these limitations, our study has several important strengths. First, we were able to identify and assess the effect of a range of side effects, including some, such changes to sexual experience and physical discomfort, which have not been extensively studied in the literature. Additionally, we used longitudinal data, following up with women one year after the baseline survey, enabling us to assess how these specific side effects related to discontinuation and switching independently, providing additional insight into contraceptive behaviors.

Women do not experience generalized "side effects and health concerns"; they experience specific side effects and contraceptive behavior is influenced based on both the side effect and on the woman's unique circumstances and context. Identifying which side effects are commonly experienced and strongly associated with discontinuation and switching, in addition to identifying some of the socio-demographic characteristics that may be associated with these behaviors, has valuable programmatic purposes. Counseling that incorporates discussions about individualized suitability for specific side effects and more discussion about the possibility of switching methods when side effects do occur may improve continuation rates. Additional research should explore in greater detail the role that context, including partner support, plays in the decision to stop or switch methods.

\section{Conclusion}


Greater attention should be paid to understanding the unique contributions of side effects to contraceptive behavior using quantitative data. We show that not all side effects are associated with contraceptive discontinuation and switching, despite the experience of side effects being common. Beyond changes to the menstrual cycle, the experience of physical discomfort and of changes to sexual experience, both of which have been largely ignored in other research, were shown to affect use. Providing greater individualized care that includes information about common side effects, while simultaneously addressing common myths and misperceptions, will better meet the needs of women.

\section{Declarations}

\section{Ethics approval and consent to participate}

All study protocols were approved by the Makerere University and Johns Hopkins University Bloomberg School of Public Health Institutional Review Boards. All participants provided oral consent to participate in the study.

\section{Consent for publication}

Not applicable

\section{Availability of data and materials}

The datasets supporting the conclusions of this article are available at www.pmadata.org. All data are available upon request.

\section{Competing interests}

The authors declare that they have no competing interests

\section{Author's contributions}

LZ conceptualized the research question, led analysis, and was responsible for drafting the manuscript. DS assisted in all stages of conceptualization, analysis, and writing. CK and SW were major contributors to interpretation of results and writing. SA advised on all aspects of study design, analysis, and provided substantial edits to the manuscript. FM and SK led study implementation, provided interpretation of results, and substantive edits to the manuscript. All authors read and approved the final manuscript.

\section{Funding}

This research was funded by the Bill and Melinda Gates Foundation (grant OPP1163880). The funders had no role in the design of the study, collection, analysis, interpretation of data, orin writing the manuscript.

\section{Acknowledgements}


The authors thank Caroline Moreau for helpful comments and suggestions and Amy Tsui for supervision and oversite.

\section{References}

1. Castle S, Askew I. Contraceptive discontinuation:reasons, challenges, and solutions. Washington, DC: Population Council and FP2020; 2015 p. 41.

2. Bradley SEK, Schwandt HM, Khan S. Levels, trends, and reasons for contraceptive discontinuation [Internet]. Calverton, Maryland, USA: ICF Macro; 2009. Available from: http://dhsprogram.com/pubs/pdf/AS20/AS20.pdf

3. Gezginc DK, Balci O, Karatayli R, Colakoglu MC. Contraceptive efficacy and side effects of Implanon ${ }^{\circledR}$. The European Journal of Contraception \& Reproductive Health Care. 2007 Jan 1;12(4):362-5.

4. Lawrie TA, Helmerhorst FM, Maitra NK, Kulier R, Bloemenkamp K, Gülmezoglu AM. Types of progestogens in combined oral contraception: effectiveness and side-effects. Cochrane Database of Systematic Reviews [Internet]. 2011 [cited 2020 Mar 27];(5). Available from: https://www.cochranelibrary.com/cdsr/doi/10.1002/14651858.CD004861.pub2/abstract

5. Kaunitz AM. Long-acting injectable contraception with depot medroxyprogesterone acetate. American Journal of Obstetrics and Gynecology. 1994 May 1;170(5, Part 2):1543-9.

6. Reid RL, Fortier MP, Smith L, Mirkin S, Grubb GS, Constantine GD. Safety and bleeding profile of continuous levonorgestrel $90 \mathrm{mcg} /$ ethinyl estradiol $20 \mathrm{mcg}$ based on 2 years of clinical trial data in Canada. Contraception. 2010 Dec 1;82(6):497-502.

7. Harper CC, Rocca CH, Darney PD, von Hertzen H, Raine TR. Tolerability of levonorgestrel emergency contraception in adolescents. American Journal of Obstetrics and Gynecology. 2004 Oct 1;191(4):1158-63.

8. Sivin I, Mahgoub SE, McCarthy T, Mishell DR, Shoupe D, Alvarez F, et al. Long-term contraception with the levonorgestrel $20 \mathrm{mcg} /$ day $(\mathrm{LNg} \mathrm{20})$ and the Copper T 380Ag intrauterine devices: A five-year randomized study. Contraception. 1990 Oct 1;42(4):361-78.

9. Sanders SA, Graham CA, Bass JL, Bancroft J. A prospective study of the effects of oral contraceptives on sexuality and well-being and their relationship to discontinuation. Contraception. 2001;64(1):51-8.

10. Tiihonen M, Leppänen H-M, Heikkinen A-M, Ahonen R. Hormonal Contraceptive Users' Self-Reported Benefits, Adverse Reactions, and Fears in 2001 and 2007. Patient-Patient-Centered-Outcome-Res. 2008 Jul 1;1(3):173-80.

11. Polis $C B$, Hussain R, Berry A. There might be blood: a scoping review on women's responses to contraceptive-induced menstrual bleeding changes. Reproductive Health [Internet]. 2018 Dec [cited 2018 Sep 28];15(1). Available from: https://reproductive-healthjournal.biomedcentral.com/articles/10.1186/s12978-018-0561-0 
12. Wood SN, Karp C, Zimmerman L. Women's sexual experiences as a side effect of contraception in low- and middle-income countries: evidence from a systematic scoping review. Sex Reprod Health Matters. 2020 Dec;28(1):1763652.

13. Campbell M, Sahin-Hodoglugil NN, Potts M. Barriers to fertility regulation: a review of the literature. Stud Fam Plann. 2006 Jun;37(2):87-98.

14. Uganda Bureau of Statistics (UBOS), ICF. Uganda Demographic and Health Survey 2016. Kampala, Uganda and Rockville, Maryland, USA: UBOS and ICF; 2018.

15. Makerere University, School of Public Health at the College of Health Sciences, Bill and Melinda Gates Institute for Population and Reproductive Health at the Johns Hopkins Bloomberg School of Public Health. Performance Monitoring and Accountability 2020 (PMA2020) Survey Round 6, PMA2018/Uganda-R6. Uganda and Baltimore, Maryland, USA; 2018.

16. Diamond-Smith N, Campbell M, Madan S. Misinformation and fear of side-effects of family planning. Culture, Health \& Sexuality. 2012 Apr;14(4):421-33.

17. Schwarz J, Dumbaugh M, Bapolisi W, Ndorere MS, Mwamini M-C, Bisimwa G, et al. "So that's why I'm scared of these methods": Locating contraceptive side effects in embodied life circumstances in Burundi and eastern Democratic Republic of the Congo. Soc Sci Med. 2019;220:264-72.

18. Bawah AA, Akweongo P, Simmons R, Phillips JF. Women's Fears and Men's Anxieties: The Impact of Family Planning on Gender Relations in Northern Ghana. Studies in Family Planning. 1999;30(1):5466.

19. Hyttel M, Rasanathan JJK, Tellier M, Taremwa W. Use of injectable hormonal contraceptives: diverging perspectives of women and men, service providers and policymakers in Uganda. Reprod Health Matters. 2012 Dec;20(40):148-57.

20. Jain A, Reichenbach L, Ehsan I, Rob U. "Side effects affected my daily activities a lot": a qualitative exploration of the impact of contraceptive side effects in Bangladesh. Open Access $\mathrm{J}$ Contracept. 2017;8:45-52.

21. Darroch JE, Sedgh G, Ball H. Contraceptive Technologies: Responding to Women's Needs. :52.

22. Kibira SPS, Muhumuza C, Bukenya JN, Atuyambe LM. "I Spent a Full Month Bleeding, I Thought I Was Going to Die..." A Qualitative Study of Experiences of Women Using Modern Contraception in Wakiso District, Uganda. PLoS One [Internet]. 2015 Nov 2 [cited 2020 May 14];10(11). Available from: https://www.ncbi.nlm.nih.gov/pmc/articles/PMC4629884/

23. Cristobal I, Lete LI, Viuda E de la, Perulero N, Arbat A, Canals I. One year quality of life measured with SEC-QoL in levonorgestrel 52 mg IUS users. Contraception. 2016 Apr;93(4):367-71.

24. Wong RC, Bell RJ, Thunuguntla K, McNamee K, Vollenhoven B. Implanon users are less likely to be satisfied with their contraception after 6 months than IUD users. Contraception. 2009 Nov 1;80(5):452-6.

25. Stephenson J, Shawe J, Panicker S, Brima N, Copas A, Sauer U, et al. Randomized trial of the effect of tailored versus standard use of the combined oral contraceptive pill on continuation rates at 1 year. Contraception. 2013 Oct 1;88(4):523-31. 
26. Rosenberg MJ, Waugh MS, Burnhill MS. Compliance, counseling and satisfaction with oral contraceptives: a prospective evaluation. Fam Plann Perspect. 1998 Apr;30(2):89-92, 104.

27. Cetina TECD, Canto P, Luna MO. Effect of counseling to improve compliance in Mexican women receiving depot-medroxyprogesterone acetate. Contraception. 2001 Mar 1;63(3):143-6.

28. Davie JE, Walling MR, Mansour DJ, Bromham D, Kishen M, Fowler P. Impact of patient counseling on acceptance of the levonorgestrel implant contraceptive in the United Kingdom. Clin Ther. 1996 Feb;18(1):150-9.

29. Capurchande R, Coene G, Schockaert I, Macia M, Meulemans H. "It is challenging... oh, nobody likes it!": a qualitative study exploring Mozambican adolescents and young adults' experiences with contraception. BMC Womens Health [Internet]. 2016 Jul 30 [cited 2018 Sep 17];16. Available from: https://www.ncbi.nlm.nih.gov/pmc/articles/PMC4967333/

30. Abdel-Tawab N, RamaRao S. Do improvements in client-provider interaction increase contraceptive continuation? Unraveling the puzzle. Patient Educ Couns. 2010 Nov 12;81(3):381-7.

31. Barden-O'Fallon J, Speizer I. What Differentiates Method Stoppers from Switchers? Contraceptive Discontinuation and Switching Among Honduran Women. International Perspectives on Sexual and Reproductive Health. 2011 Mar;37(01):016-23.

32. Korn EL, Graubard BI. Epidemiologic studies utilizing surveys: accounting for the sampling design. Am J Public Health. 1991 Sep;81(9):1166-73.

33. StataCorp. Stata Statistical Software: Release 16. College Station, TX: StataCorp LLC; 2019.

34. von Elm E, Altman D, Egger M, Pocock S, Gotzsche P, Vandenbroucke J. The Strengthening the Reporting of Observational Studies in Epidemiology (STROBE) Statement: guidelines for reporting observational studies.

35. Morse JE, Rowen TS, Steinauer J, Byamugisha J, Kakaire O. A qualitative assessment of Ugandan women's perceptions and knowledge of contraception. International Journal of Gynecology \& Obstetrics. 2014;124(1):30-3.

36. Callahan RL, Brunie A, Mackenzie ACL, Wayack-Pambè M, Guiella G, Kibira SPS, et al. Potential user interest in new long-acting contraceptives: Results from a mixed methods study in Burkina Faso and Uganda. PLOS ONE. 2019 May 28;14(5):e0217333.

37. Sedlander E, Bingenheimer JB, Thiongo M, Gichangi P, Rimal RN, Edberg M, et al. "They Destroy the Reproductive System": Exploring the Belief that Modern Contraceptive Use Causes Infertility. Studies in Family Planning. 2018;49(4):345-65.

38. Gueye A, Speizer IS, Corroon M, Okigbo CC. Belief in Family Planning Myths at the Individual And Community Levels and Modern Contraceptive Use in Urban Africa. Int Perspect Sex Reprod Health. 2015 Dec;41(4):191-9.

39. Dumbaugh M, Bapolisi W, Bisimwa G, Mwamini M-C, Mommers P, Merten S. Navigating fertility, reproduction and modern contraception in the fragile context of South Kivu, Democratic Republic of Congo: "Les enfants sont une richesse." Cult Health Sex. 2019;21(3):323-37. 
40. Higgins JA, Smith NK. The Sexual Acceptability of Contraception: Reviewing the Literature and Building a New Concept. The Journal of Sex Research. 2016 May 3;53(4-5):417-56.

41. Higgins JA, Kramer RD, Ryder KM. Provider Bias in Long-Acting Reversible Contraception (LARC) Promotion and Removal: Perceptions of Young Adult Women. Am J Public Health. 2016 Sep 15;106(11):1932-7.

42. Diedrich JT, Desai S, Zhao Q, Secura G, Madden T, Peipert JF. Association of short-term bleeding and crampingpatterns with long-acting reversible contraceptive method satisfaction. American Journal of Obstetrics and Gynecology. 2015 Jan 1;212(1):50.e1-50.e8.

43. Kalton G, Schuman H. The Effect of the Question on Survey Responses: A Review. Journal of the Royal Statistical Society Series A (General). 1982;145(1):42-73.

44. Friborg $\mathrm{O}$, Rosenvinge $\mathrm{JH}$. A comparison of open-ended and closed questions in the prediction of mental health. Qual Quant. 2013 Apr 1;47(3):1397-411.

\section{Figures}




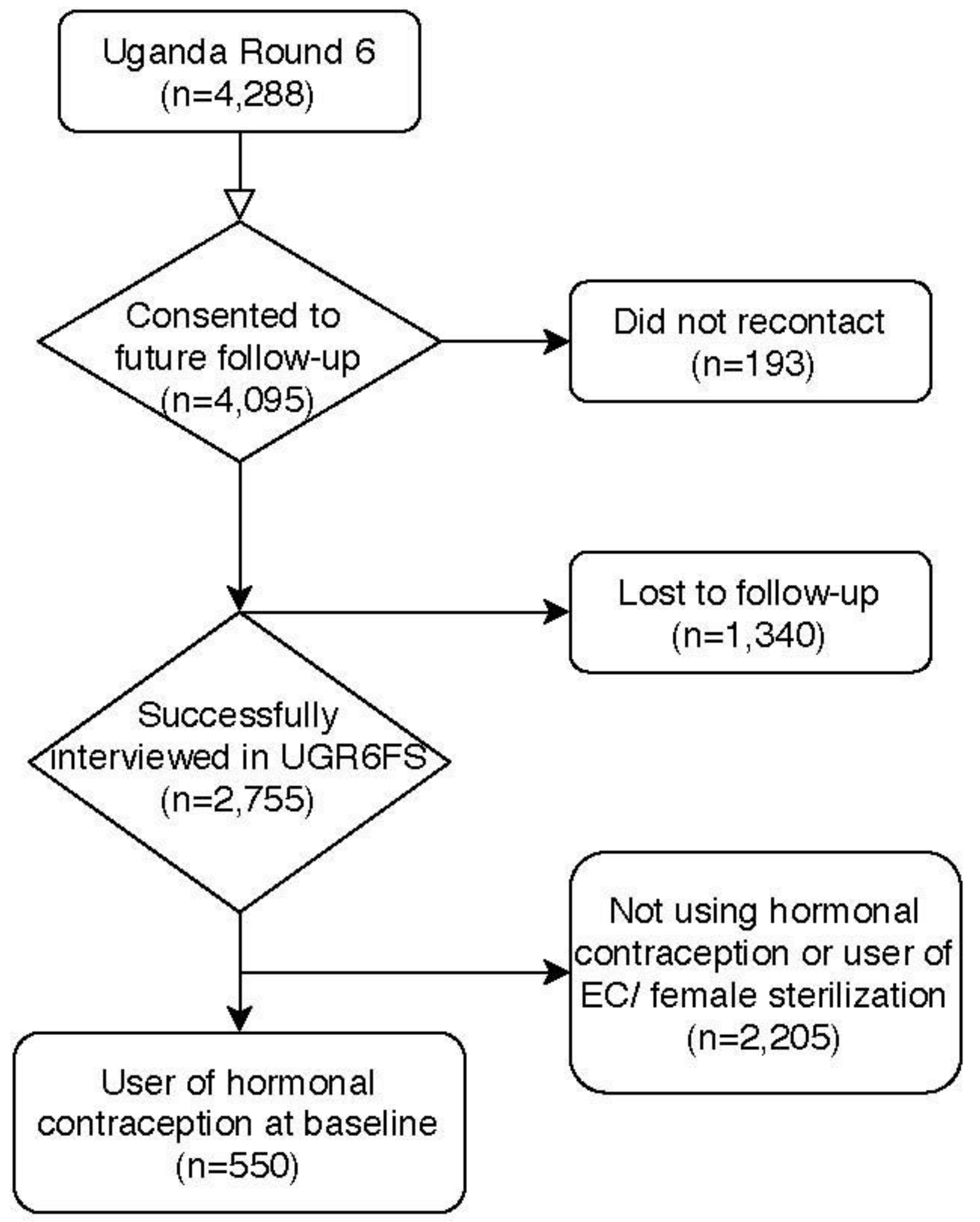

Figure 1

Selection Process for Analytic Sample

\section{Supplementary Files}

This is a list of supplementary files associated with this preprint. Click to download. 
- completedSTROBEcohortchecklist.docx 